\title{
Seguimiento y análisis de los conocimientos de Seguridad industrial en los estudiantes de Grado en Ingeniería Química: influencia del uso de metodologías activas y realización de prácticas en empresa
}

\section{B. Garcia-Fayos ${ }^{\mathrm{a}}$, Maria Sancho Fernández ${ }^{\mathrm{b}}$ y Jose Miguel Arnal Arnal $^{\mathrm{c}}$}

aUniversitat Politècnica de València. Departamento de Ingeniería Química y Nuclear. E-mail: $\underline{\text { beagarfa@iqn.upv.es }}{ }^{\mathrm{b}} \underline{\text { msanchof } @ \text {,iqn.upv.es }}{ }^{\mathrm{c}}$ jarnala@iqn.upv.es

\begin{abstract}
Industrial safety is a key tool for the development of the professional activity of the Chemical Engineering graduates. Its teaching and learning should be integrated into the curriculum of the degree in a transverse or specific way, and should encourage reasoning and critical thinking as well as finding solutions to real security problems through practical activities and the implementation of active methodologies. This paper analyzes the security knowledge acquired by students of Chemical Engineering in a short and medium term and the influence of the use of active methodologies, progressive learning or internship practices as tools for improving the results. Evolution is analyzed through two fourth-year courses called Industrial Processes and Industrial Safety. The results show that the combination of active learning methodologies along with a guided teaching increases the level of results as well as an increase in working time or reinforcement of knowledge through several courses in different academic courses. Finally, the student motivation is essential in improving the results because allowsdevelopment of effective learning skills for their professional life.
\end{abstract}

Keywords: Industrial Safety, Industrial Processes, Chemical Engineering, Active methodologies, diagnostic evaluation.

\footnotetext{
Resumen

La seguridad industrial es una herramienta clave para el desarrollo de la actividad profesional del graduado en Ingeniería Química. Su enseñanzaaprendizaje debe ser integrada en el curriculum de la titulación bien de forma transversal o especifica, y debe fomentar el razonamiento y pensamiento crítico así como la búsqueda de soluciones a problemas de seguridad reales a través de actividades prácticas y la aplicación de metodologías activas. El presente trabajo analiza los conocimientos de seguridad adquiridos por los alumnos de Ingeniería Química a corto y medio plazo así como la influencia del uso de metodologías activas, aprendizaje progresivo o realización de prácticas en empresa como herramientas para la mejora de los resultados obtenidos. Se analiza la evolución a través de dos asignaturas de cuarto curso Procesos industriales y Seguridad Industrial. Los resultados muestran que la combinación de metodologías activas junto con un aprendizaje guiado incrementa significativamente el nivel de los resultados así como el aumento del tiempo de trabajo o el refuerzo de los conocimientos a través de
} 
Seguimiento y análisis de los conocimientos de Seguridad industrial en los estudiantes de Grado en Ingeniería Química: influencia del uso de metodologías activas y realización de actividades en empresas

asignaturas en distintos cursos académicos. Por último, resulta fundamental la motivación de los alumnos en la mejora de los resultados obtenidos ya que permite un aprendizaje efectivo de competencias para su vida profesional.

Palabras clave: Seguridad industrial, Procesos industriales, Ingeniería Química, Metodologías activas, evaluación de diagnóstico.

\section{Introducción}

La Seguridad industrial es una formación clave para los futuros profesionales de la Ingeniería Química dado que es una herramienta para prevenir accidentes en la industria y minimizar las graves consecuencias de carácter económico, medioambiental y humano que estos provocan. El ingeniero químico a través de su actividad profesional debe ser capaz de identificar en los procesos y en el entorno de trabajo los agentes físicos, químicos y biológicos que suponen un riesgo, evaluar el nivel de riesgo de esta exposición y establecer medidas preventivas que reduzcan el nivel riesgo asociado en una planta o actividad industrial a niveles tolerables (Darbra, 2012).

Existe un común acuerdo en afirmar que la enseñanza en seguridad debe ser obligatoria en todos los cursos de Ingeniería Química (Pitt, 2012; Shallcross, 2013a) y que debe ser considerada, no como un complemento de la enseñanza de procesos industriales, sino como una parte integral del curriculum de la ingeniería (Hendershot, 2007), y que debe ser impartida de forma sistemática (Perrin, 2008), coherente y coordinada.

Las asociaciones de Ingenieros químicos internacionales coinciden en que la enseñanza sobre seguridad debe incluir (Meyer, 2015):

-Técnicas para la motivación real de los estudiantes en una contínua educación en seguridad después de su graduación y durante toda la vida.

-Métodos de resolución activos y también conceptos teóricos de seguridad.

-Enseñanza sobre las consecuencias de las negligencias en seguridad.

En relación a los conceptos, (Bryan, 1999 y Shallcross 2013b), sugieren que los graduados en Ingeniería química deben al menos adquirir competencias sobre los siguientes contenidos:

-Encontrar información sobre seguridad y salud y específicamente normativa y legislación

-Derechos y responsabilidades del empleado y del empleador en relación a la ley cuando estan desempeñando su trabajo

-Elaboración de informes y recogida de registros

-Lucha contra incendios: prevención y protección

-Peligros asociados al manejo de productos químicos, material tóxico y residuos peligrosos

-Permisos y procedimientos de trabajo, especialmente para trabajo en espacios confinados

(cc) EY-NC-ND 2016, Universitat Politècnica de València 
-Gestión de sistemas de seguridad

-Operación y respuesta frente a emergencias, tales como vertido de productos peligrosos

-Factor humano y su influencia en la seguridad

-Peligros asociados al mantenimiento y puesta en marcha

-Control procesos

-Diseño seguro

El objetivo final es formar profesionales capaces de identificar, seleccionar, evaluar y comparar distintas alternativas para resolver problemas de seguridad durante su vida laboral de forma independiente y óptima. Por ello se precisa una enseñanza-aprendizaje basado en el razonamiento (Ferjencik, 2007) que fomente el papel activo de los alumnos de construcción de su propio aprendizaje y su transferencia a situaciones reales (De Miguel, 2006), acercándose de esta forma al rol profesional que desarrollarán. Para ello, resulta fundamental el uso de metodologías activas que fomenten el pensamiento creativo sobre seguridad, así como la identificación de alternativas, comparación, selección y evaluación de éstas para la búsqueda de soluciones a problemas concretos (Darbra, 2012; Ferjencik, 2007).

\section{Objetivos}

El objetivo de este trabajo es analizar el aprendizaje a corto y medio plazo sobre Seguridad industrial de los estudiantes de Grado en Ingeniería Química de la Universitat Politècnica de València a través de las evidencias recogidas en los puntos de control establecidos (al finalizar una asignatura troncal de cuarto curso cuatrimestre A, y al comenzar una asignatura optativa de cuarto curso cuatrimestre B) de los tres últimos cursos académicos.

Asimismo, se analizarán los resultados obtenidos teniendo en cuenta los cambios de metodología realizados en la asignatura así como la influencia de la realización de prácticas en empresa de los alumnos y de la formación transversal sobre seguridad realizada en asignaturas previas.

\section{Contexto y desarrollo de la innovación educativa}

El trabajo se ha realizado en dos asignaturas de cuarto de Grado en Ingeniería Química, en concreto en Procesos Industriales (PIIQ) de la Ingeniería Química (troncal de 4.5 creditos ECTS) y en Seguridad Industrial (SI) (optativa de la mención "Diseño y Seguridad industrial" de 4.5 ECTS) durante los cursos académicos 2013-2014, 2014-2015 y 2015-2016.

La asignatura de Seguridad Industrial al ser optativa implica que, aproximadamente, solo un tercio de los alumnos de Ingeniería Química reciben formación específica sobre seguridad. Por ello, se decidió integrar esta materia de forma transversal a través de distintas asignaturas troncales tanto teóricas como experimentales con el fin de trabajar distintos contenidos fundamentales para los futuros profesionales.

En el curso 2013-2014, se impartió por primera vez la asignatura de Procesos Industriales en la titulación (Garcia-Fayos, 2016) y se decidió introducir (también por primera vez para los alumnos) contenidos de seguridad en la asignatura. En concreto a través de una práctica de aula guiada y una lección magistral, los alumnos y el profesor analizaban e interpretaban la información de una ficha de seguridad de un producto químico. Una parte de estos alumnos, 
Seguimiento y análisis de los conocimientos de Seguridad industrial en los estudiantes de Grado en Ingeniería Química: influencia del uso de metodologías activas y realización de actividades en empresas

cursaron también la asignatura de Seguridad Industrial (Garcia-Fayos, 2015), que se impartía en el cuatrimestre B, y también por primera vez en la titulación de Ingeniería Química. En este caso se optó por una metodología tradicional, basada en lecciones magistrales y resolución de problemas, complementadas con una visita de campo a empresa.

Dado que los resultados académicos en la asignatura de Procesos Industriales y en la de Seguridad Industrial no fueron satisfactorios, se decidió realizar algunas modificaciones tanto en los contenidos como en la metodología de ambas asignaturas en el curso académico 20142015.

En la asignatura de Procesos industriales, el tema de la seguridad se planteó con los mismos contenidos que el curso anterior pero a través de una práctica de aula en la que los alumnos tenían un papel más autónomo y en la que debían interpretar información sobre la ficha de seguridad de un producto químico. Esto fue posible porque en el curso anterior los alumnos ya habían recibido formación sobre seguridad y salud en las asignaturas de tercer curso previas experimentales Experimentación en Ingeniería Química II (EIQII) y Experimentación en Ingeniería Química III (EIQIII) (Arnal, 2013; Sancho, 2015), por lo que se pudieron reforzar contenidos impartidos en las asignaturas previas e introducir contenidos nuevos. Además se duplicó el tiempo dedicado a este tema en la planificación de la asignatura.

Finalmente en el curso 2015-2016, en la asignatura de Procesos Industriales se introdujo el estudio de casos de accidentes reales como metodología activa y el trabajo cooperativo, dado que los alumnos ponían en común las causas de varios accidentes reales acontecidos en la industria química a través de una presentación oral en grupo y voluntaria. Por su parte, la asignatura de Seguridad industrial se mantuvo con la metodología del curso anterior.

La Fig 1, resume el contexto de la innovación educativa y la introducción de la formación en Seguridad en tercer y cuarto curso del Grado en Ingeniería Química. 

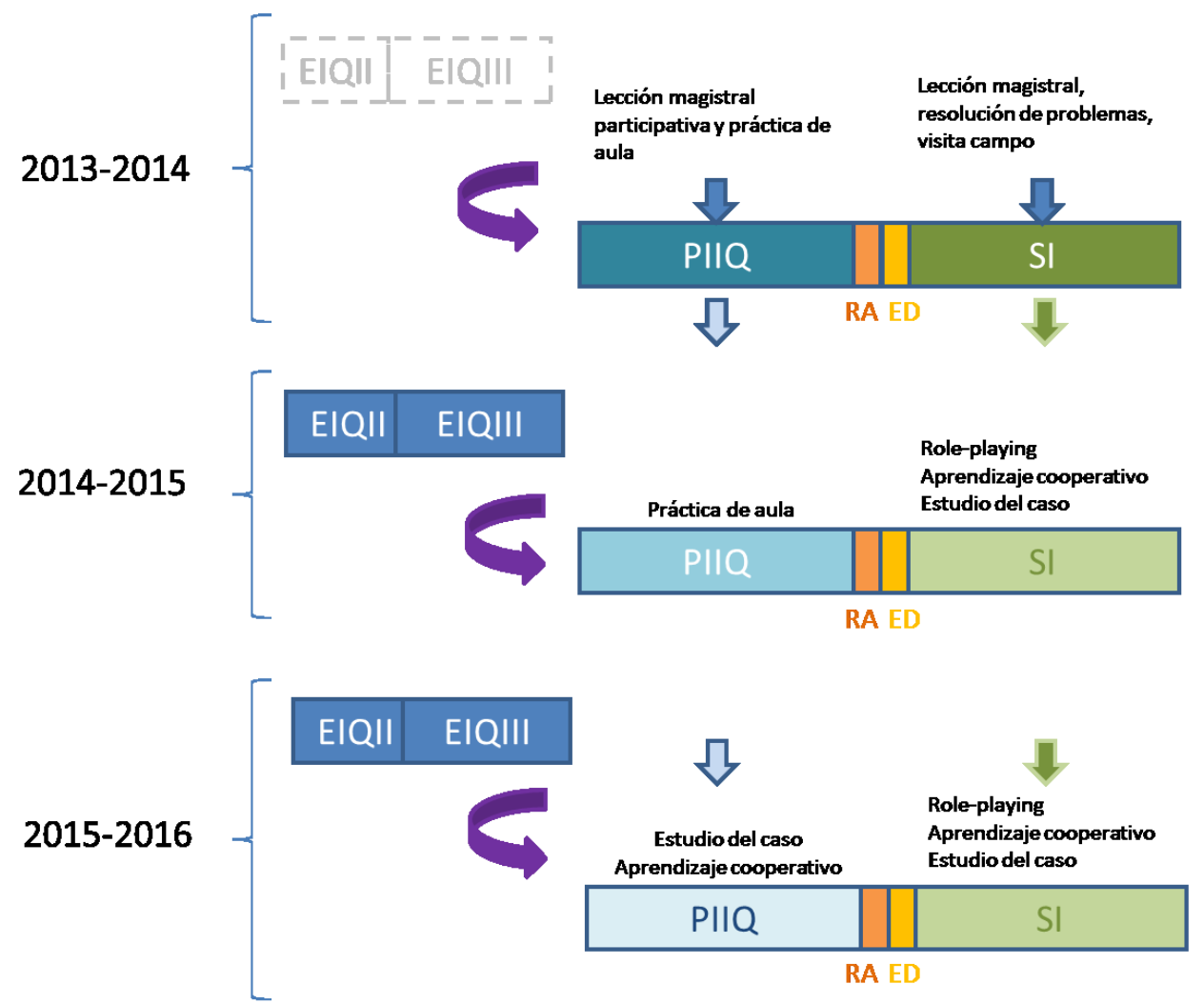

Fig. 1 Introducción de la formación en Seguridad en el grado en Ingeniería Química en la UPV

Para evaluar la influencia que los cambios de metodología han tenido sobre el aprendizaje a corto y medio plazo en Seguridad industrial de los alumnos de grado en Ingeniería Química se han utilizado las siguientes herramientas:

-Encuesta de diagnóstico(ED): En la primera sesión de clase de la asignatura Seguridad Industrial se pasó una encuesta anónima a los alumnos con tres bloques diferenciados:

-Bloque I: Contenidos sobre seguridad industrial no tratados previamente en ninguna asignatura de la titulación (seguridad, riesgo, extracción localizada, máquinas-herramientas, amianto, equipos a presión) a través de 8 preguntas

-Bloque II: Contenidos relacionados con los impartidos en las asignaturas experimentales de Experimentación en Ingeniería Química II y III de tercer curso (reglamento REACH, frases $\mathrm{H}$ y $\mathrm{P}$, ficha de seguridad, pictogramas de productos químicos y su significado) a través de 4 preguntas

-Bloque III: Contenidos relacionados con la asignatura de Procesos Industriales impartidos en el cuatrimestre anterior (Electricidad estática y medidas de prevención en trasvase y almacenamiento y propiedades físico-químicas de sustancias inflamables) a través de 3 preguntas

Además, los alumnos, debían responder si estaban realizando o habían realizado prácticas en empresa y si habían cursado previamente o no la asignatura de Procesos industriales en Ingeniería Química.

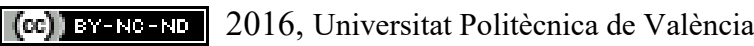

Congreso In-Red (2016) 
Seguimiento y análisis de los conocimientos de Seguridad industrial en los estudiantes de Grado en Ingeniería Química: influencia del uso de metodologías activas y realización de actividades en empresas

La encuesta fue cumplimentada por los alumnos matriculados en la asignatura: 5 alumnos en el curso 2013-2014, 14 alumnos en el curso 2014-2015 y 9 alumnos en el curso 2015-2016 y la calificación de los bloques se promedió y transformó a base 10 .

-Resultados académicos (RA): se ha analizado la calificación obtenida en la pregunta sobre seguridad de la prueba escrita de respuesta abierta de la asignatura de Procesos Industriales realizada por los alumnos al finalizar la asignatura y que pretende evaluar los resultados a corto plazo. En el curso 2013-2014 cumplimentaron el examen 53 alumnos, en el curso 20142015, 69 alumnos y en el curso 2015-2016, 99 alumnos, cifras que se corresponden con el $100 \%$ de los alumnos matriculados en la asignatura. Se ha tenido en cuenta para el análisis de los resultados los alumnos que posteriomente cursaron la asignatura de Seguridad Industrial con el fin de poder diferenciar y comparar el promedio global de la muestra con el de este grupo específico.

\section{Resultados}

A continuación se analizan los resultados recogidos a través de las herramientas comentadas en el apartado anterior.

\subsection{Resultados de la evaluación de diagnóstico realizada en Seguridad Industrial}

La Fig 2. muestra los resultados de la evaluación de diagnóstico previa realizada en la asignatura de Seguridad Industrial. Se muestran los resultados para los tres cursos académicos y para cada uno de los bloques de preguntas. La nota de cada bloque aparece como un promedio de las respuestas obtenidas y en base 10 para facilitar la comparación.

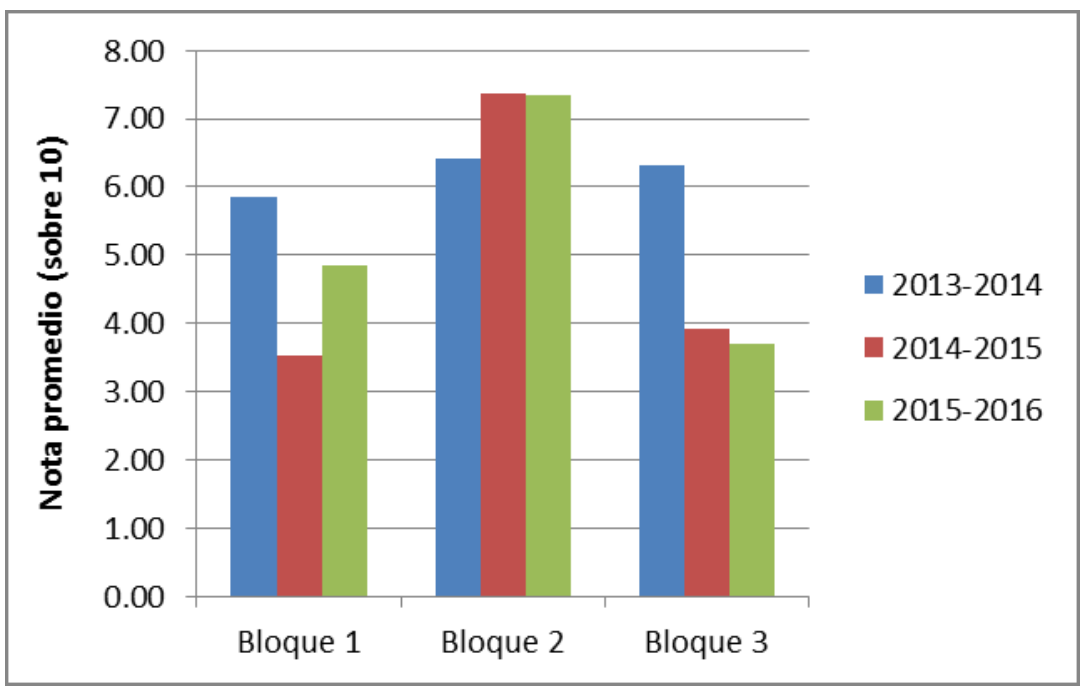

Fig. 2 Resultados de la evaluación de diagnóstico previa en Seguridad Industrial

En primer lugar se observa que los resultados son mejores para los alumnos del curso 20132014 en todos los bloques, excepto en el bloque 2. Esta inferior calificación en el bloque 2 de los alumnos del curso 2013-2014 es debida a que los alumnos de ese curso, no recibieron formación previa sobre seguridad y salud en las asignaturas de experimentación previas y

(cc) EY-NC-ND 2016, Universitat Politècnica de València

Congreso IN-RED (2016) 
todos los contenidos relacionados con este bloque fueron vistos por primera vez en la asignatura de Procesos industriales durante ese curso, previa a la de Seguridad Industrial. Por el contrario, los alumnos de los cursos 2014-2015 y 2015-2016, realizaron los seminarios sobre seguridad y salud en las asignaturas de experimentación previas (1 curso académico antes) y reforzaron estos mismos contenidos en la asignatura de Procesos Industriales. Este refuerzo se refleja claramente en una calificación superior en los contenidos reforzados, al evaluarlos en la encuesta a través del Bloque 2 (calificación casi 1 punto superior). La menor calificación de los resultados en el bloque 3, de los cursos 2014-2015 y 2015-2016 (en promedio por debajo de 4) respecto del curso 2013-2014 (en promedio superior al 6), resulta conveniente analizarla. En este resultado puede influir si se ha cursado la asignatura de Procesos previamente y/o la realización de prácticas en empresa, pero sobretodo los cambios de metodología que se han llevado a cabo en la asignatura de Procesos industriales durante los últimos tres años para impartir la seguridad. Se da la circunstancia que los resultados en este bloque son opuestos a los obtenidos en los exámenes en promedio en los últimos tres años, en los que la nota más alta se ha obtenido en el curso 2015-2016 (como se verá psoteriomente en la Fig. 4). No obstante, también conviene analizar la nota en seguridad del examen de Procesos de la muestra compuesta por los alumnos que eligieron después cursar la asignatura de seguridad por si hubiera diferencias. Todo estos aspectos se analizarán en el apartado 4.3.

Por último, se observa que el bloque 1 es el más fluctuante en cuanto a sus notas promedio. Esto es debido a que se trata de un bloque que evalúa contenidos no trabajados en las asignaturas analizadas, por lo que su conocimiento puede haberse adquirido bien por curiosidad o interés personal, a través de noticias o por la experiencia profesional previa adquirida por ejemplo a través de prácticas en empresa. Por ello, en el apartado siguiente se analizará la influencia de la experiencia profesional en la adquisición de conocimientos relacionados con la seguridad.

\subsection{Analisis de la influencia de las prácticas en empresa para la adquisición de nuevos conocimientos en Seguridad industrial}

La Fig. 3 muestra los resultados del bloque 1 de la encuesta de diagnóstico realizada en seguridad industrial en los cursos 2014-2015 y 2015-2016, diferenciando entre los alumnos que realizaron prácticas en empresa y los que no. 
Seguimiento y análisis de los conocimientos de Seguridad industrial en los estudiantes de Grado en Ingeniería Química: influencia del uso de metodologías activas y realización de actividades en empresas

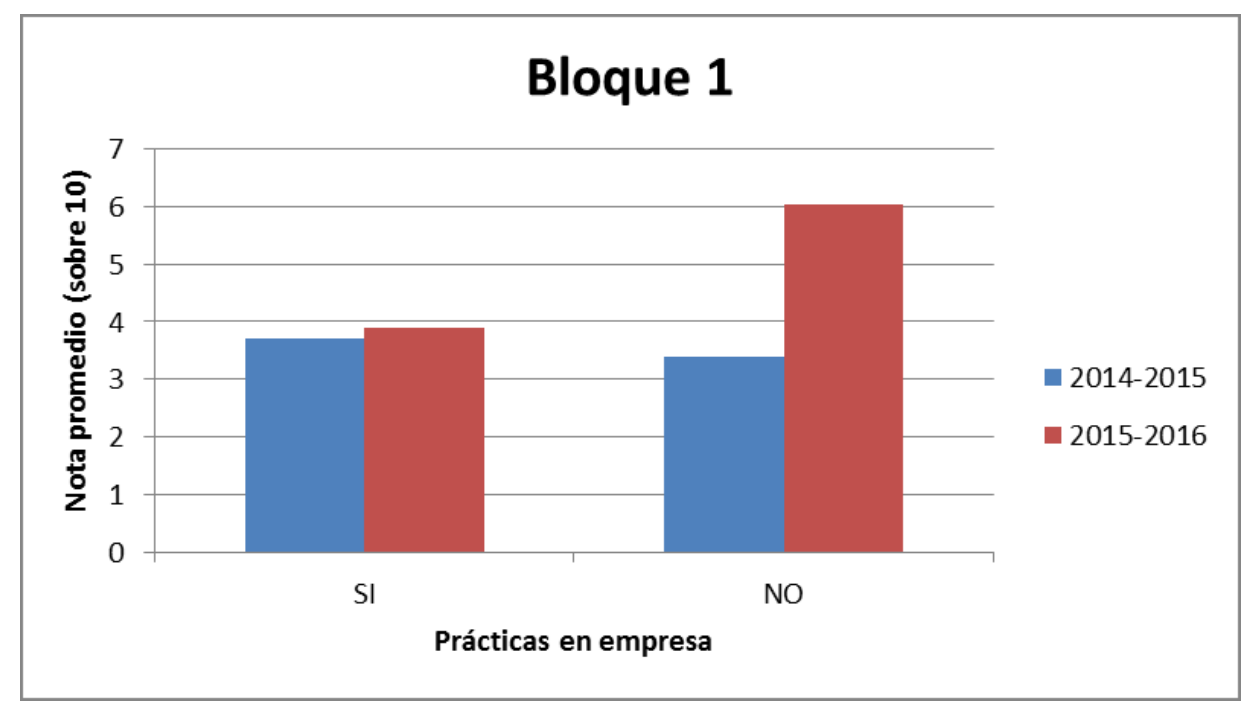

Fig. 3 Resultados del bloque 1 de la encuesta de Seguridad Industrial e influencia de las prácticas en empresa

Para el análisis de esta variable, solo se tienen los datos de los cursos 2014-2015 y 20152016. Conviene recordar que los contenidos del bloque 1, no se han impartido previamente en las asignaturas relacionadas, y por tanto no es esperable que el alumno supere estas preguntas.

Como se observó previamente en la Fig. 2, para el curso 2014-2015 se obtuvo que el promedio es de 3.52 sobre 10 y para el curso 2015-2016 el promedio fue de 4.84 sobre 10 , lo que implica que en ambos casos no se alcanzó la puntuación mínima de 5 puntos sobre 10 para considerarlo como suficiente.

Dicho esto, si se diferencian las respuestas obtenidas en función de si realizaron prácticas en empresa o no, los resultados varían ligeramente. En la figura anterior se observa que, para el curso 2014-2015, apenas hay diferencia entre el nivel de las respuestas de los alumnos que han realizado prácticas en empresas y los que no, obteniéndose un resultado promedio similar. Para el curso 2015-2016, hay una diferencia promedio de 2.5 puntos, entre los que han realizado prácticas en empresa y los que no, mejorando los resultados para los alumnos que no realizaron prácticas en empresa.

Esta diferencia puede deberse al tipo de prácticas realizadas y si estaban relacionadas o no con la Seguridad Industrial, al tipo de empresa y su actividad productiva asi como el trabajo asignado al alumno y el momento en que se realizaron las prácticas (ese mismo cuatrimestre, el cuatrimestre anterior, el curso anterior). A la vista de los resultados obtenidos, resulta interesante para los cursos siguientes, incluir estas variables en las encuestas de recogida de resultados realizadas a los alumnos, con el fin de poder analizar esta variable con mayor profundidad.

4.3 Analisis del aprendizaje a medio plazo de los contenidos impartidos en la asignatura de Procesos industriales

(cc) EY-NC-ND 2016, Universitat Politècnica de València 
En este apartado se muestra la apropiación de los conocimientos adquiridos por los alumnos a medio plazo, impartidos en Procesos Industriales y evaluados de nuevo en la encuesta de diagnóstico realizada en Seguridad Industrial 4 meses después. Conviene aclarar que a pesar de que se trata de asignaturas que se imparten de forma correlativa entre cuatrimestres, los conceptos de seguridad en la asignatura de Procesos Industriales se imparten en el periodo docente que transcurre de Septiembre a finales de Octubre (primer parcial) y la asignatura de Seguridad se inicia en Febrero. La Fig. 4 diferencia los resultados en promedio de los que cursaron previamente Procesos Industriales y los que no, en los dos cursos académicos 20142015 y $2015-2016$.

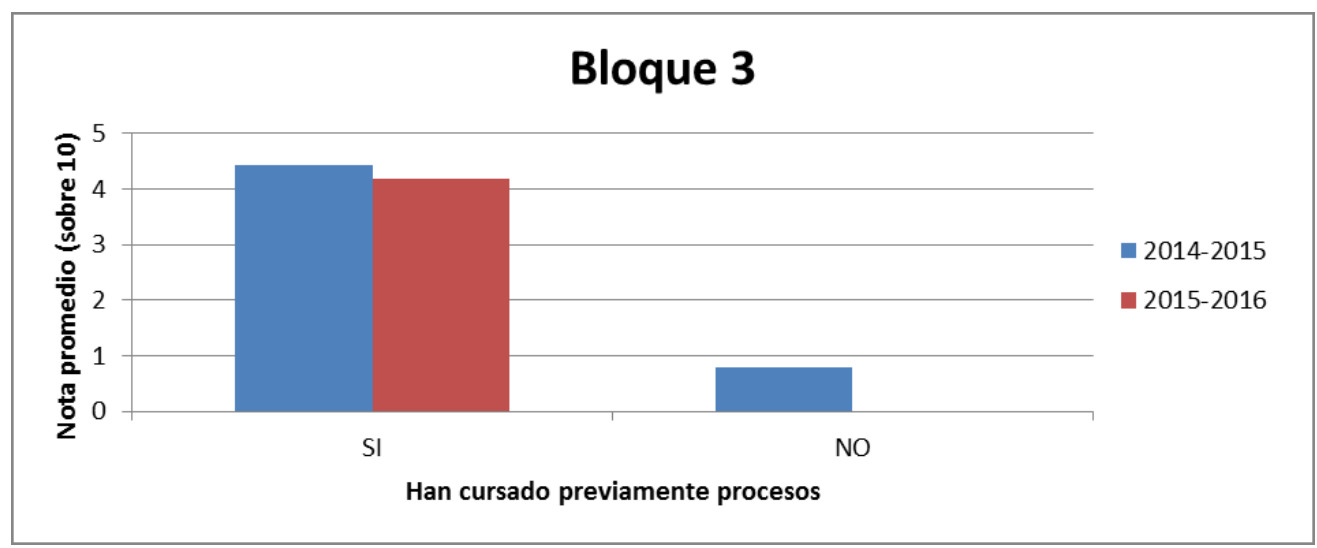

Fig. 4 Resultados del bloque 3 de la encuesta de Seguridad Industrial e influencia de cursar la asignatura de Procesos Industriales

En primer lugar se observa que la nota promedio en todos los casos es baja y está situada por debajo de 5 puntos sobre 10 .

Dicho esto, se observa que los alumnos que han cursado y superado la asignatura previa de "Procesos industriales en la Ingeniería Química", poseen mayor conocimiento de los contenidos que los que no han cursado la asignatura, como era esperable. Cabe destacar que esta encuesta se ha realizado 4 meses después de cursar la asignatura, por lo que valora los conocimientos adquiridos y asimilados por los alumnos, que perdurán a través del tiempo.

Por otra parte, si se quiere analizar la evolución de estos conocimientos a lo largo del tiempo, se puede comparar los resultados promedio obtenidos en el examen de la asignatura de Procesos industriales con los de la encuesta de diagnóstico realizada 4 meses después. En el análisis de los resultados del examen se diferenciará entre el promedio obtenido por todos los alumnos y el obtenido por el grupo de alumnos que posteriomente cursarán la asignatura de Seguridad Industrial. 
Seguimiento y análisis de los conocimientos de Seguridad industrial en los estudiantes de Grado en Ingeniería Química: influencia del uso de metodologías activas y realización de actividades en empresas

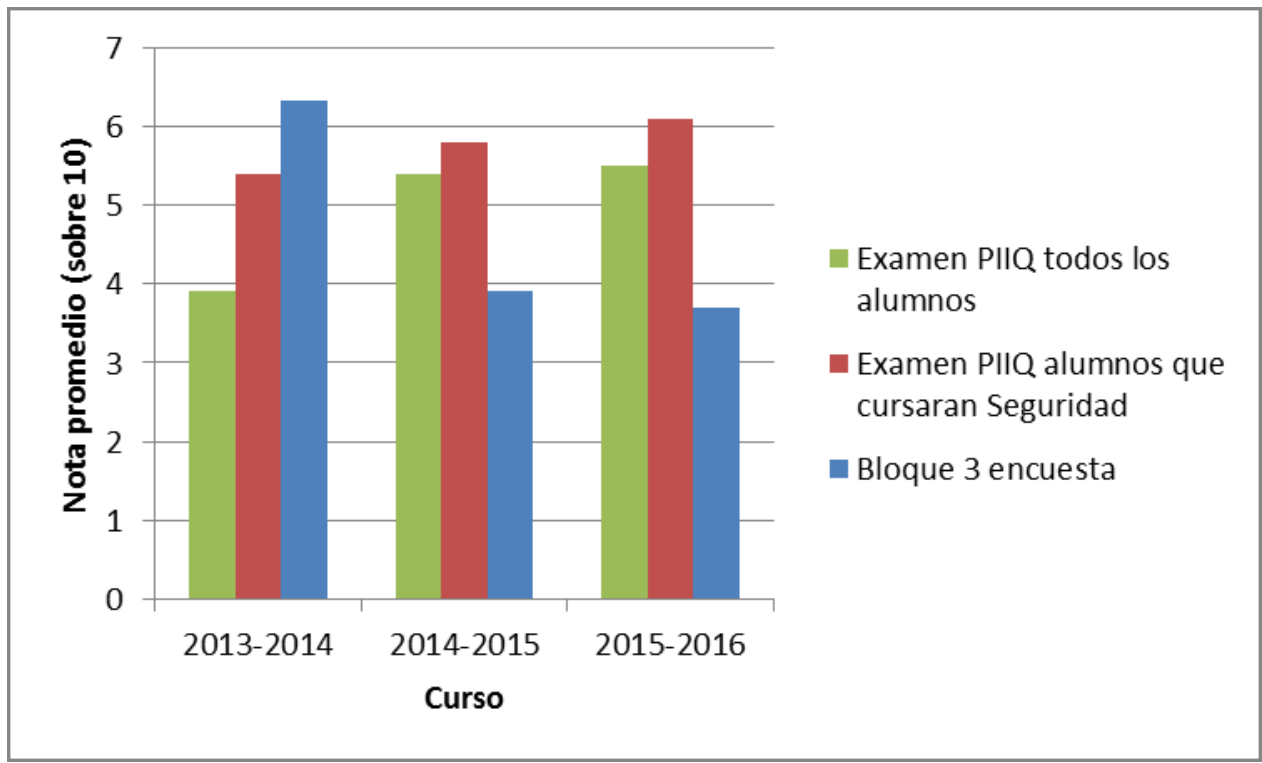

Fig. 4 Comparación de los resultados del examen de Procesos Industriales y del bloque 3 de la encuesta de Seguridad industrial

En la Fig. 4 se observa que para el curso 2013-2014, las notas de los exámenes son más bajas que en el caso de la encuesta realizada 4 meses después, mientras que para los cursos 20142015 y 2015-2016 los resultados de los exámenes son más altos que los de la encuesta de diagnóstico. Además se observa que en los tres cursos académicos, los resultados promedio del examen de los alumnos que posteriomente iban a cursar Seguridad son más altos que el promedio del conjunto de alumnos, lo que indicaría que su mayor motivación e interés por este tema (dado que todos realizaron el trabajo voluntario de seguridad en la asignatura de Procesos y que posteriomente han elegido voluntariamente cursar una asignatura optativa específica de Seguridad), les permite alcanzar unos mejores resultados en el examen cuando se evalua esta materia en la asignatura troncal de Procesos Industriales.

La diferente tendencia entre los cursos 2013-2014 y 2014-2015/2015-2016 en relación al examen y encuesta será analizada a continuación. En el curso 2013-2014, se utilizó una metodología mixta entre lección magistral y práctica de aula que produjo resultados poco satifactorios en el examen (promedio de 3.9) y que planteó un cambio de metología como se explicó previamente en el apartado de Contexto y motivación de la innovación educativa. En 2014-2015, se dio más protagonismo al alumno en la práctica de aula guiada y se duplicó el tiempo dedicado a la seguridad, obteniendo resultados considerablemente mejores en el examen, incrementándose en promedio 1.5 puntos sobre 10. Con el fin de seguir mejorando estos resultados, en el curso 2015-2016 se mantuvo el tiempo dedicado a la Seguridad pero se cambió a una metodología de trabajo cooperativo y presentación oral basado en el análisis de casos reales de fallos de seguridad, con el fin de aumentar más la motivación de los alumnos al ver las implicaciones de estos fallos y al tratarse de casos reales de la industria química. Sin embargo, aunque la calificación de los trabajos voluntarios fue elevada 
(promedio de 8.4 sobre 10 ), los resultados en el examen se mantuvieron y apenas mejoraron respecto del curso anterior.

Por tanto, parece que el cambio de metodología en la asignatura de Seguridad Industrial (especialmente el aumento del tiempo dedicado al desarrollo de los temas realizado a partir del curso 2014-2015) sirvió para mejorar los resultados obtenidos en el examen de la asignatura, pero no tuvo tanta influencia sobre los conocimientos de seguridad asimilados a medio plazo, como evidencian los bajos resultados obtenidos en el bloque 3 de la encuesta previa de seguridad de la asignatura de Seguridad industrial.

El uso de metologías activas para trabajar la seguridad ha mejorado los resultados a corto plazo pero no tanto los evaluados a medio plazo, donde parece que la combinación de una metodología más formal (lección magistral) junto con una metodología activa (práctica de aula guiada) ha permitido que los alumnos interioricen mejor los contenidos tras 4 meses impartidos. En futuros trabajos, será necesario analizar si esto es debido a que se requiere una mayor madurez e implicación del alumnado así como mayor tiempo trabajo en el aula y cierto conocimiento previo en seguridad del alumnado para lograr un mayor éxito con el uso de estas metodologías en el ámbito de la seguridad industrial.

\section{Conclusiones}

Las principales conclusiones de este trabajo son las siguientes :

-Los contenidos en Seguridad trabajados y reforzados en tres asignaturas troncales y consecutivas de la titulación permiten obtener excelentes resultados en cuanto a nivel de aprendizaje de los alumnos, tanto a corto como a medio plazo.

-La motivación e interés personal o profesional de los alumnos sobre la seguridad mejora el nivel de los resultados obtenidos permitiendo un aprendizaje efectivo y la adquisición de competencias en materia de seguridad que el alumno necesita aplicar en su vida profesional real.

-El aumento del tiempo dedicado al trabajo de la seguridad en el aula ha demostrado ser el factor más influyente a la hora de mejorar el nivel de los resultados de aprendizaje de los alumnos, al menos, a corto plazo.

-La combinación de metodologías activas y enseñanza formal por el profesor permite obtener mejores resultados en la formación en seguridad a medio plazo que el uso exclusivo de metodologías activas.

-Los resultados sobre la realización de prácticas en empresa no han sido concluyentes para analizar su utilidad como herramienta en la adquisición de nuevos conocimientos relacionadas con la seguridad industrial.

-Para los siguientes cursos académicos se propone analizar también los conocimientos iniciales de seguridad en la asignatura de Procesos industriales previamente a plantear el aprendizaje cooperativo y el estudio del caso, con el fin de valorar la necesidad de realizar alguna práctica guiada de seguridad antes de la realización de este trabajo con el fin de mejorar los resultados obtenidos tanto a corto como a medio plazo.

(cc)) BY-NC-ND 2016, Universitat Politècnica de València

Congreso In-Red (2016) 
Seguimiento y análisis de los conocimientos de Seguridad industrial en los estudiantes de Grado en Ingeniería Química: influencia del uso de metodologías activas y realización de actividades en empresas

\section{Referencias}

ARNAL, J.M., SANCHO, M., GARCÍA-FAYOS, B. (2013). "Integration of industrial hygiene and safety in experimental subjects of chemical engineering degree". IATED. En: 7 th International Technology, Education and Development Conference INTED 2013 (marzo, 2013, Valencia). Valencia: Editorial IATED Academy

BRYAN, L.A. (1999). "Educating engineers on safety” en Journal of Management in Engineering,15, 30 .

DARBRA, R.M.; CASAL, J.; PASTOR, E., VILCHEZ J.A.; ARNALDOS, J.; PLANAS E. (2012). "Risk analysis active learning through the investigation of real cases" en Process Safety and Environmental Protection, 90, 430.

DE MIGUEL M. “Modalidades de enseñanza centradas en el desarrollo de competencias”. Universidad de Oviedo (2006).

FERJENCIK, M. (2007). "Best starting point to comprehensive process safety education" en Process Safety Progress, Vol. 26 (3), pp. 195-202.

GARCÍA-FAYOS, B., SANCHO, M. y ARNAL, J. M. (2015). “Aplicación de metodologías activas de aprendizaje en una nueva asignatura de Grado en Ingeniería Química". Instituto de Ciencias de la Educación ICE-UPV. En: Congreso Nacional de Innovación Educativa y Docencia en Red IN-RED15 (julio, 2015, Valencia). Valencia: Editorial Universitat Politècnica de València.

GARCÍA-FAYOS, B., SANCHO, M. y ARNAL, J. M. (2016). “Teaching and learning industrial safety in chemical engineering: cooperative work based on the analysis of case studies of accidents in the chemical industry". IATED. En: 10 th International Technology, Education and Development Conference INTED 2016 (marzo, 2016, Valencia). Valencia: Editorial IATED Academy.

HENDERSHOT, D.C. y SMADES W.(2007). "Safety culture begins in the classroom" en Process Safety Progress, Vol. 26 (2), pp. 83-84.

MEYER, T. (2015) "Towards the implementation of a safety educationprogram in a teaching and research institution". Education for chemical engineers (2015). DOI:10.1016/j.ece.2015.06.003

PERRIN, L., LAURENT A. (2008). "Current Situation and future implementation of safety curricula for chemical engineering education in France" en Education for Chemical Engineers, 3, e84.

PITT, M.J. (2012). “Teaching safety in Chemical Engineering. What, How and Who?" en Chemical Engineering and Technology, 35, 8, 1341.

SANCHO, M., GARCIA-FAYOS B., J.M. ARNAL (2015). "Tracking of safety learning in two last years of chemical engineering degree" ". En: 9 th International Technology, Education and Development Conference INTED 2015 (marzo, 2015, Madrid). Madrid: Editorial IATED Academy

SHALLCROSS, D.C. (2013a). "Safety shares in the chemical engineering classroom" en Education for Chemical Engineers, 9, e94.

SHALLCROSS, D.C. (2013b). "Safety education through case study presentations" en Education for Chemical Engineers, 8, e12. 
B. Garcia-Fayos, M. Sancho Fernandez, J.M. Arnal Arnal

(c) EY-NC-ND 2016, Universitat Politècnica de València

Congreso In-Red (2016) 